\title{
Violation of Bell Inequalities as a Violation of Fair Sampling in Threshold Detectors
}

\author{
Guillaume Adenier \\ International Center for Mathematical Modeling in Physics Engineering, Economy and Cognitive Sc. \\ University of Växjö, Vejdes Plats 6, 35195 Växjö, Sweden
}

\begin{abstract}
Photomultiplier tubes and avalanche photodiodes, which are commonly used in quantum optic experiments, are sometimes referred to as threshold detectors because, in photon counting mode, they cannot discriminate the number of photoelectrons initially extracted from the absorber in the detector. We argue that they can be called threshold detectors on more account than that. We point out that their their functioning principle relies on two thresholds that are usually thought unimportant individually in the context of EPR-Bell discussion. We show how the combined effect of these threshold can lead to a significant sampling selection bias in the detection of pairs of pulses, resulting in an apparent violation of Bell inequalities.
\end{abstract}

\section{Introduction}

As much as EPR-Bell experiments have consistently shown results in agreement with the predictions of quantum mechanics, they have however never been able to entirely remove the possibility of local realistic models explaining the observed correlations. Various loopholes that render the probability space explicitly contextual have been identified, and it is the concern of experimenters to make sure that these loopholes cannot be responsible for the observed violation of Bell inequalities. The derivation of a Bell inequality [1] requires indeed that an experiment involving several incompatible measurements can be written on a single probability space, independently of the measurement context. This has been criticized by a number of authors [2, 3, 4, 5, 6], and is at the heart of the Växjö Interpretation of Quantum Mechanics [7, 8, 9, 10, 11, 12, 13, 14, 15]-which is a contextual statistical realistic interpretation of Quantum Mechanics.

The main class of contextualizing loopholes that have been thoroughly addressed in EPR experiments are the ones based on a communication between the parties. By rapidly changing the measurement settings during the flight of the particles, experimenters have excluded the possibility of an exchange of information between the parties through a classical channel. The first experiment to thus enforce a space-like interval between the remote measurements was that of Aspect et al [16] by means of a periodical and asynchronous switching between the measurement settings. The concept was furthered by Weihs et al [17] with a random fast switching, and it was extended recently to separating the measurement settings from the emission at the source by a space-like interval [18].

The other main class of contextualizing loopholes arises when an experiment is performed with a low overall efficiency 1. The correlations are therefore not measured on the population of all emitted pairs, as a derivation of a Bell inequality would have it, but on a small sample instead. The trouble occurs if the sampling process itself is unfair, that is, if the probability for a given particle to be detected in either channel depends on the local measurement setting. Then the subset $\Omega_{\alpha}$ (resp. $\Omega_{\beta}$ ) of the sample space $\Omega$ that is spanned locally by the single events detected by Alice (resp. by Bob) depends explicitly on the local measurement context $\alpha$ (resp. $\beta$ ). At first sight, such a contextuality might appear harmless since it is fully local. However, the events involved in a Bell inequality test are paired events detected in coincidence, which means that the subset of the sample space spanned in the experiment becomes the intersection 2 of the two local subsets, that is $\Omega_{\alpha \beta}=\Omega_{\alpha} \cap \Omega_{\beta}$. So, the combination of two local unfair sampling processes together with the coincidence condition yields a global contextuality: the sample space $\Omega_{\alpha \beta}$ associated to the measurement depends explicitly on the entire measurement context, a feature that is known to compromise the derivation of Bell inequality [2, 14]. It is interesting to note that the contextuality of the entire measurement setup arises here by construction when Bohr had only posited it in his answer [20] to the challenge to the completeness of quantum mechanics by Eintein, Podolski and Rosen [21].

\footnotetext{
${ }^{1}$ An experiment with ion traps [19] did reach perfect detection efficiency. It could not however fulfill the locality condition and was thus powerless to challenge local realism the way EPR-Bell experiments are meant to.

${ }^{2} \mathrm{~A}$ pair is counted as such if both Alice AND Bob register a click simultaneously.
} 
Local realistic models exploiting this detection loophole have long been proposed [22], and have been discussed many times since [23, 24, 25, 26, 27, 28, 29, 30, 31, 32], but in the absence of any physical justification for the introduction of such a bias, the existing models using this loophole are usually deemed to be ad hoc [22, 27, 28].

We would like to argue that the detectors used in EPR experiments might very well be responsible for a sample selection bias due to a threshold effect in their detection capability. Photomultiplier tubes and avalanche photodiodes, which are commonly used in quantum optic experiments, are sometimes referred to as threshold detectors [33] because they cannot discriminate the number of photons striking a detector simultaneously. We would like to argue that these detectors can be called threshold detectors on more account than that. It is known that there is a fundamental limit on the minimum detectable pulse energy for a given signal-to-noise ratio[34, 35, 36], and contrary to what is sometimes asserted [37], we would like to argue that photomultiplier tubes and avalanche photodiodes rely in their functioning principle on thresholds as well, and that it is a feature that should carefully be taken into account in the framework of quantum information in general, in particular in the case of a violation of Bell inequalities.

\section{Apparent violation of Bell inequalities with a simple threshold detector}

To illustrate how the existence of a threshold in detectors can be relevant to the violation of Bell inequalities, let us consider the simplest possible model of a threshold detector within a classical framework.

We consider first a source sending pulses impinging on a polarizing beam splitter (PBS) with its main axis oriented along the direction $\phi$. Each pulse is assumed to carry the same energy $E_{0}$, and the polarization of the pulse is set by a random variable $\lambda$ uniformly distributed on the interval $[0,2 \pi]$. Two threshold detectors are set at the two outputs of the PBS (reflected and transmitted). Let $\Phi$ be the detection threshold. A click is recorded if the portion of the pulse $E$ reaching the detector is greater than this threshold $\Phi$.

By Malus law, the energy reaching the detectors (+) and (-) at the output of the PBS is:

$$
\begin{aligned}
& E^{+}=E_{0} \cos ^{2}(\lambda-\phi)=\frac{1+\cos 2(\lambda-\phi)}{2} \\
& E^{-}=E_{0} \sin ^{2}(\lambda-\phi)=\frac{1-\cos 2(\lambda-\phi)}{2}
\end{aligned}
$$

The measurement results are then:

- In the (+) detector channel:

- $A^{+}=+1$ if $E^{+}>\Phi$, that is, $\cos 2(\lambda-\phi)<\frac{2 \Phi}{E_{0}}-1$

- $A^{+}=0$ otherwise

- In the (-) channel:

- $A^{-}=+1$ if $E^{-}>\Phi$, that is, $\cos 2(\lambda-\phi)<-\left(\frac{2 \Phi}{E_{0}}-1\right)$

$-A^{-}=0$ otherwise

Double clicks occur when both the (+) and (-) channel record a click simultaneously (for the same pulse), that is, when

$$
\frac{2 \Phi}{E_{0}}-1<\cos 2(\lambda-\phi)<-\left(\frac{2 \Phi}{E_{0}}-1\right)
$$

We can impose that no double clicks are recorded, by which we mean that at most one of the output channel of the PBS can record a click for each input pulse. This can be interpreted as placing ourselves in a 'single photon regime' in the framework of threshold detectors, at least if we stick to the minimalist maxim attributed to Zeilinger [38]: "Photons are clicks in photon detectors.". We will see that this condition is fundamental to guarantee an apparent violation of Bell inequalities, not only with this simple model, but with more complex models of threshold detectors as well. This condition is realized by imposing that the lower bound in the previous equation is greater than the upper bound $\frac{2 \Phi}{E_{0}}-1>-\left(\frac{2 \Phi}{E_{0}}-1\right)$, or

$$
\frac{\Phi}{E_{0}}>\frac{1}{2}
$$

Naturally, we also have the condition $\Phi<E_{0}$ if we want any click to be recorded at all. 


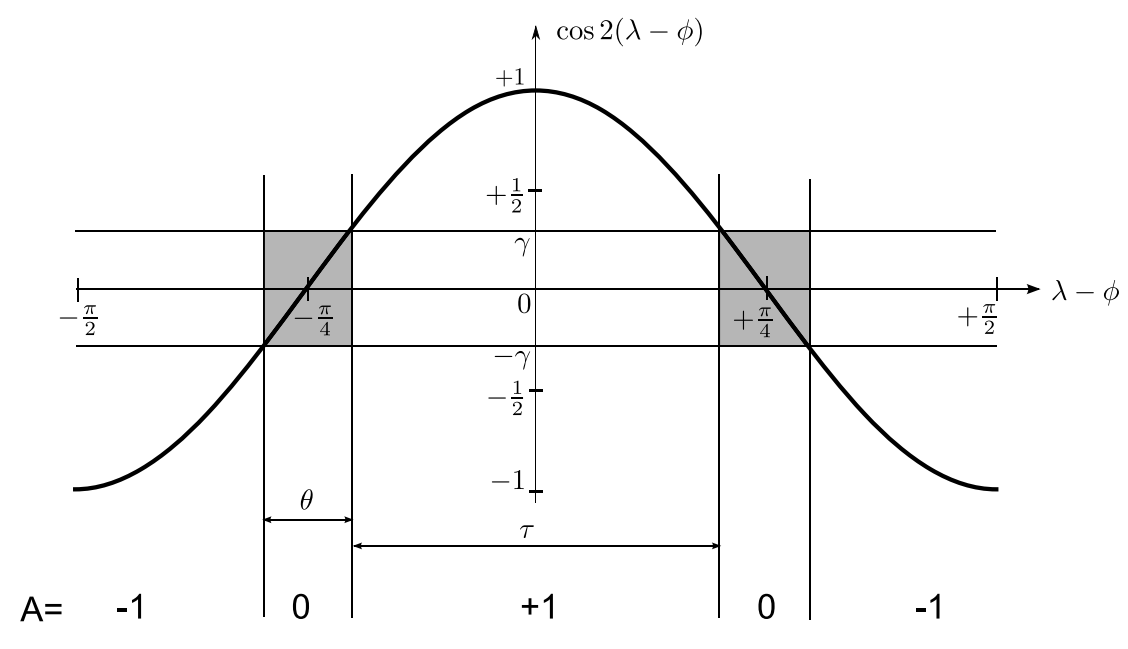

Figure 1: Measurement result as a function of $(\lambda-\phi)$.

To summarize the behavior of the polarimeter consisting of a PBS and its two output detectors, we define

$$
\gamma=\frac{\Phi}{E_{0}}-\frac{1}{2}
$$

with $0<\gamma<\frac{1}{2}$.

The measurement results are displayed in Fig. 1 and can be written:

- $A=+1$ if $\cos 2(\lambda-\phi)>\gamma$

- $A=-1$ if $\cos 2(\lambda-\phi)<-\gamma$

- $A=0$ if $-\gamma<\cos 2(\lambda-\phi)<\gamma$.

Let $\theta$ be the size of the non detection region. We have $A=0$ when $\arccos (-\gamma)<2(\lambda-\phi)<\arccos (\gamma)$, that is,

$$
\theta=\frac{1}{2}(\arccos (-\gamma)-\arccos (\gamma))=\arcsin (\gamma)
$$

with the periodicity condition $\tau+\theta=\pi / 2$, where $\tau$ is the size each detection region.

We now consider pairs of pulses sent towards two such polarimeters controlled by Alice and Bob, in a typical EPRB configuration. We require that the pulses are correlated in polarization, that is $\lambda_{1}=\lambda_{2}=\lambda$, with identical energy $E_{0}$. We label $\phi_{1}$ and $\phi_{2}$ the measurement settings of the polarimeters controlled by Alice and Bob respectively, with $\Delta \phi=\phi_{1}-\phi_{2}$.

We can write the correlation as a piecewise function, depending on the parameter $\Delta \phi$ :

- $0<|\Delta \phi| \leq \theta$ :

$$
\begin{aligned}
& P^{++}(\Delta \phi)=P^{--}(\Delta \phi)=2(\tau-\Delta \phi) \\
& P^{+-}(\Delta \phi)=P^{-+}(\Delta \phi)=0
\end{aligned}
$$

The correlation function is then constant:

$$
E=\frac{4(\tau-\Delta \phi)}{4(\tau-\Delta \phi)}=1
$$

- $\theta<|\Delta \phi| \leq \tau$ :

$$
\begin{aligned}
& P^{++}(\Delta \phi)=P^{--}(\Delta \phi)=2(\tau-\Delta \phi) \\
& P^{+-}(\Delta \phi)=P^{-+}(\Delta \phi)=2(\Delta \phi-\theta)
\end{aligned}
$$

The correlation function decreases linearly with $\Delta \phi$ :

$$
E=\frac{4(\tau-\Delta \phi)-4(\Delta \phi-\theta)}{4(\tau-\theta)}=\frac{-2 \Delta \phi+\tau+\theta}{\tau-\theta} .
$$




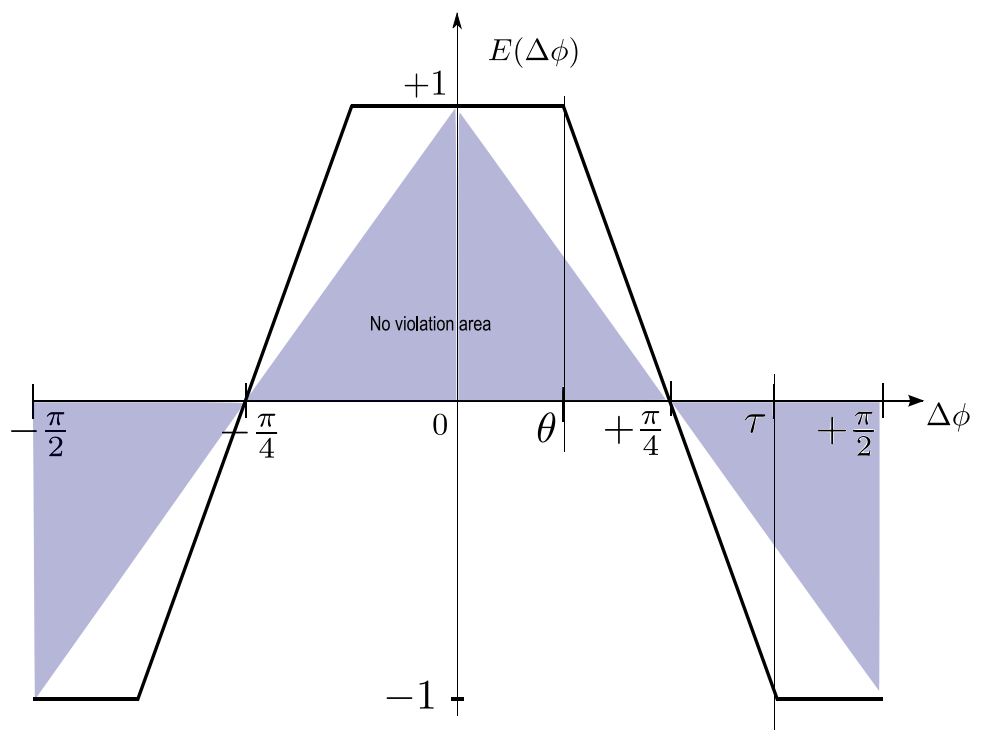

Figure 2: The correlation function gives an apparent violation of Bell's inequality. The shaded area represent Bell's bound: outside this area a violation of Bell inequality is possible for a well chosen set of measurement settings.

- $\tau<|\Delta \phi| \leq \tau+\theta$ :

$$
\begin{aligned}
& P^{++}(\Delta \phi)=P^{--}(\Delta \phi)=0 \\
& P^{+-}(\Delta \phi)=P^{-+}(\Delta \phi)=2(\tau-\theta)+2(\Delta \phi-\tau)
\end{aligned}
$$

The correlation function is then constant:

$$
E=-\frac{4(\Delta \phi-\theta)}{4(\Delta \phi-\theta)}=-1
$$

The resulting correlation function is displayed in Fig. 2 It is always greater than Bell's bound, and thus exhibits the appearance of a violates Bell's inequality. The apparent violation is due to the fact that the probability of joint detection depends on the angle difference $\Delta \phi$, so that even though the number of joint detection decreases for small $\Delta \phi$, the correlation function remains unchanged and of magnitude 1 for small angle difference (modulo $\pi / 2$ ): it has the appearance of a stronger correlation than would be classically possible, but it is of course only apparent and due to an biased (unfair) sampling process.

For instance, if $\theta>\frac{\pi}{8}$, then $E\left(|\Delta \phi|=\frac{\pi}{8}\right)=1, E\left(|\Delta \phi|=\frac{3 \pi}{8}\right)=-1$ and the CHSH function yields:

$$
\begin{aligned}
S & =\left|E\left(0, \frac{\pi}{8}\right)+E\left(0,-\frac{\pi}{8}\right)+E\left(\frac{\pi}{4}, \frac{\pi}{8}\right)-E\left(\frac{\pi}{4},-\frac{\pi}{8}\right)\right| \\
& =4 .
\end{aligned}
$$

The correlation function as displayed in Fig. 2 is admittedly still far from the result predicted by Quantum Mechanics, but as we will see below, a slightly more sophisticated modelization of the threshold detectors can lead to an observed correlation function in very good agreement with those predictions.

\section{Photomultipliers and photodiodes as threshold detectors}

Photomultipliers and photodiodes come as a large variety of detectors, with different absorbing materials and designs, but we would like nevertheless to sketch the characteristics that they have in common and that are important for our analysis. In particular, we would like to stress that these detectors rely on two fundamental thresholds, that we will call the energy band threshold and the discriminator threshold.

\subsection{Work function and Energy band threshold}

The working principle of photomultipliers and avalanche photodiodes rely on the excitation of energy carriers bound in a potential well: in photomultipliers, the energy carriers are electrons that are extracted from the metal absorber to 
the vacuum, whereas in avalanche photodiodes carriers are electron-hole pairs, the electrons being extracted from the valence band to the conduction band.

The detection principle of a photomultipliet 3 is that electrons are bound in the absorbing material by a potential well, and the trigger that ultimately leads to a measurable current in the detector is an electron eventually overcoming this potential barrier. It is the finite size of the potential well binding the electron that constitutes this first threshold; the energy transmitted to the electron has to be high enough to free the electron from its potential well. In terms of energy band, an electron susceptible to overcome this potential well is in a low energy band, where it is susceptible to absorb an incoming radiation. It has to overcome a band gap in order to reach a higher energy band (the vacuum) where it can be accelerated enough to let other electrons overcome the band gap as well. The minimum required energy is called the work function $\Phi$. This first threshold operates at the input of the detector and can be referred to as the band gap threshold. It implies that such a threshold detector is practically blind to any incoming radiation constituted of particles with energy less than that of the work function. This feature is in fact very useful as it allows these detectors to be operational for low light signals, even at room temperature. It prevents the detector from being blinded by the many fluctuations at lower frequencies than the targeted signal frequency. This energy threshold was interpreted by Einstein by quantizing the electromagnetic field and associating an energy $h \nu$ to a light quanta of frequency $\nu$. The presence of a minimum energy $\Phi$ allowing for the photoelectric effect translates into a minimum frequency $\nu_{0}$. It should however be remembered that the photoelectric effect can be explained as a resonance effect by a semiclassical treatment, in which the field is treated classically while only the energy levels in matter are quantized [39, 40]. We would therefore like to consider the energy conservation equation used by Einstein $E=\Phi+E_{\text {kmax }}$ independently of his quantizing hypothesis and consider that $E$ simply represents the energy of a the electromagnetic pulse striking the detector, and $E_{\mathrm{kmax}}$ the maximum kinetic energy imparted to the liberated electron (at zero temperature).

The principle of photoemission can be described in three sequential phases [41, 42]:

- the absorption of a radiation by an electron in the material increases its energy.

- the energized electron diffuses through the material, losing some of its energy by colliding with other electrons (electron scattering) or with the lattice (phonon scattering), and may reach the material-vacuum interface.

- the electron reaching the surface with enough excess energy with respect to the surface barrier that always exist at an interface between material and vacuum may escape from it.

Energy losses vary from material to material, but can occur in each of these steps. In metals, the losses due to electron scattering are important due to the large number of electrons in the conduction band, so that in effect the escape depth - the depth at which an electron absorbing a photon may have a good chance of reaching the surface with enough energy - is small (only a few nanometers). Electrons absorbing light at greater depth have practically no chance to reach the surface, so that increasing the thickness of the photocathode beyond the escape depth does not result into an increase of efficiency. At this thickness, photocathode are semi-transparent, so that less than half the visible light can interact with such a layer. This puts a practical limit to the quantum efficiency of photocathodes, independently of the potential barrier.

In semiconducting material, the escape depth is larger due to the low number of free electrons in the conduction band, which increases the practical efficiency of such materials.

\subsection{Dark counts and Multiplication noise: necessity of a discrimination threshold}

A second threshold, known as the discriminator threshold, arises due to the inherent noise in threshold detectors, and the necessity to decide when an output pulse is the signature of a detected signal, or when it corresponds to noise instead.

The first source of noise is the multiplication process. In photomultipliers, once an electron has been extracted from the metal, it is accelerated in vacuum by an electric field until its kinetic energy is enough to extract other bounds electrons (secondary emission) when striking the surface of another metal surface (dynode), which will in turn be used accelerated onto other dynodes to free more and more electrons, until that flow of electron $\left(\sim 10^{7}\right)$ becomes measurable as anodic current 4 .

\footnotetext{
${ }^{3}$ For simplicity, we will focus here on the case of photomultipliers to describe the detection process, and we will describe the differences or similarities in the working principle of (avalanche) photodiodes when necessary.

${ }^{4}$ In an avalanche photodiode, a reverse bias is applied to the P-N junction of the diode so that a high-field is formed in the depletion layer, where the radiation is absorbed. Energy carriers (electrons and holes) are accelerated by this field, and collide with other atoms, liberating secondary carriers which are in turn accelerated and collide with other atoms (avalanche effect).
} 
The multiplication factor is not a constant, it varies significantly from one liberated energy carrier to another. In a photomultiplier, the gain is given by

$$
g=\alpha \delta^{N}
$$

where $\delta$ is the multiplication factor of a single dynode, $\alpha$ is the fraction of photoelectrons collected by the multiplier structure, and $N$ is the number of stages in the photomultiplier. In the most simple model, $\delta$ can be assumed to follow a Poisson distribution about the average yield for each dynode, so that the gain is a compound Poisson process over $N$ identical stages. However, experimental measurements of the single photoelectron pulse height spectra from photomultipliers exhibit a distribution with larger relative variance than predicted by the Poisson model (and in some case with an decreasing exponential distribution instead of a peaked one), and there is thus no universal description of multiplication statistics [43].

The second source of noise is due to the fact that even in total darkness a current can still be measured at the output of a photomultiplier or of a photodiode. This background is referred to as the dark current, or dark counts (depending on the operating mode of the detector). It is due to various sources of identified noise, such as ohmic leakage, regenerative effects, and cosmic radiations; but the main source of noise under normal condition is thermionic emission.

As we have seen, a ionization occurs when the energy of the electron is high enough to let it overcome this potential barrier. This can naturally happen when an incoming radiation excites an energy carrier, but this can also happen through thermal fluctuation even in the absence of any incoming signal. Even though the probability of having an electron spontaneously overcoming the band gap due to thermionic energy is very small (as $k T \ll \Phi$ at room temperature), the large number of free electrons in the absorber means that this will in effect lead to a significant number of thermionic pulses in the output of the detector. The amplitude distribution of the dark current varies according to the type of dynode, and may vary considerably between different samples of the same photomultiplier. However, because a thermionic photoelectron can appear at any stage in the multiplication process, the background spectrum typically has a high proportion of undersized pulses (fractional photoelectrons).

The combination of thermionic emissions with the stochastic nature of the multiplication leads to output pulses that have a large dispersion of shape and amplitude, with an excess of small amplitude pulses.

Ideally, one would wish to be able to subtract these unwanted small pulses from the signal that is to be measured by subtracting this background from the measured signal plus background. In low light applications, the optimum sensitivity is obtained in photon counting mode. The idea is to eliminate the effect of varying pulse heights by using a discriminator. A discriminator is a circuit that produces a specified output signal if and only if it receives an input pulse whose amplitude exceeds in one case or is less than an assigned value in another case[42]. It produces a standard pulse for all photomultiplier pulses above a fixed threshold [44]. It is only when the output current is greater than this low level discrimination value that a pulse is discretized as a valid "click".

Setting the value of this discriminator threshold is a delicate point. A too low threshold will pick up many dark counts, thus degrading the signal and the correlation in coincidence counting. A higher level threshold will improve the signal-to-noise ratio, but at the cost of rejecting many low pulses, thus decreasing the efficiency of the detector [45, 46, 44]. Knowing how the efficiency of detectors is a crucial weakness in EPR-Bell experiments, the influence of this discriminator threshold should be considered with the utmost attention. When the output distribution of single photoelectron has a peak distribution, one would ideally set the threshold in the valley right before the peak, but this requires to plot a pulse height distribution and is not always practical. One possibility consists in plotting the count rate as a function of the threshold for a fixed high voltage, and chose a value on the observed plateau [45]. There is however a level of arbitrariness in this choice. For instance, Aspect [45] chose a value in the center of the plateau, with a higher discrimination that was possible, but with the advantage of stability (in the presence of variations in the multiplication voltage), whereas Grangier [46] chose a higher threshold, at the cost of lower efficiency, but with less spurious counts.

In the literature on the experimental violation of Bell inequalities, the choice of the discriminator threshold is at best discussed after the theoretical discussion, as a merely technical detail that only influences the quantum efficiency of the detector thought as black box. We believe that the discrimination threshold should be included in any discussion involving detection of weak light pulses because it can have a manifest effect on statistics that are investigated. Indeed rejecting low pulses may very well introduce a sample selection bias, favoring the appearance of non classical effects such as an apparent violation of Bell inequalities. 


\section{Simulations with classically correlated pairs of pulses}

To sum up, the two thresholds that we wish to consider in photomultipliers and photodiodes in the context of EPR-Bell experiments are:

- Energy gap threshold (by which the low energy inputs pulses are discarded)

- Discriminator threshold (by which the low amplitude outputs pulses are discarded)

We believe that the influence of these thresholds may been overlooked in the discussion on non-classical effects, and in particular in EPR and Bell inequality discussion. Individually, each threshold seems quite harmless, but we will show here that their combined effect can have a significant influence on the coincidence counting statistics. Indeed the combined effect of these two threshold opens the possibility of a fundamental, and yet tunable, detection threshold in photon counting, which can lead to a sample selection bias in the detected events, leading to an apparent violation of Bell inequalities in a fully local realistic framework.

We have performed numerical simulations in which a pair of pulses with same energy and polarization are sent over arbitrary distances. Alice and Bob make a polarization measurement using polarizing beam splitter (PBS). Two threshold detectors record the 'clicks' at the output of the two possible output (reflected and transmitted) of each PBS. They count the number of coincidences and compute the correlation function.

A source creates pairs of classical laser pulses with identical linear polarization $\lambda$ :

- The polarization of pairs is uniformly distributed over $[0,2 \pi]$.

- The polarization measurements are performed with polarizing beam spitters $\varphi_{1}$ and $\varphi_{2}$.

- The collection efficiency is set to 1 for simplicity. Each pulse is fully absorbed by an energy carrier.

- The detectors in each of the four channels are modelized with varying

- Ratio of pulse energy over the band gap threshold

- Discrimination threshold

In the simplest version of our simulation, we have used some simplifying assumptions that are quite unrealistic, but that nevertheless show the essential feature of the problem. We have assumed that each pulse carries the same energy $E_{0}$, that the pulses are well separated in time so that only one pulse reaches a detector at a time, and that the energy $E$ reaching a detector is fully absorbed by one single energy carrier. We have also assumed that the potential well binding the energy carrier has a well defined and fixed size $\Phi$, so that the energy conservation is written $E=\Phi+E_{k}$, where $E_{k}$ is the excess energy of the carrier after absorption. The multiplication was flawless (the gain $g$ was set to 1 ), so that the output current $i=g \cdot E_{k}$ and the detector was exempt of dark counts. The discriminator $D$ is a logical gate evaluating the proposition $i>D$. In the absence of noise and with a gain of 1 , increasing the discrimination threshold $D$ is simply equivalent to increasing the potential well binding the energy carriers by an amount $D$.

In order to get closer to the features of real detectors, we have considered the fact that the work function is a minimum for the potential well seen by the energy carrier, which can be modelized by introducing some losses in the absorption of the incoming energy by the carrier. The losses alone in the absorbed energy are enough to make the model flexible enough to recover the predictions of Quantum mechanics, by using non symmetrical detection pattern. This is done settings having a different ratio between the energy of the incoming pulse $E_{0}$ and the work function $\Phi$ at the two stations controlled by Alice and Bob, which effectively changes the efficiency at each station. One can then obtain detection patterns pretty similar to that of the Gisin-Larsson model [28, 27, 29] (see Fig. 3), leading to a correlation function and a total number of counts that mimics the predictions of Quantum mechanics rather nicely (see Fig .4).

By varying simultaneously the ratio $E_{0} / \Phi$, where $E_{0}$ is the energy of the incoming pulse $E_{0}$ and $\Phi$ the work function, together with the discrimination threshold $D$, we can obtain a 3D map showing for which couple of parameters an apparent violation of Bell inequalities is obtained (see Fig. 5), as well as the corresponding total number of coincidences. The result shows a significant region where an (apparent) violation of Bell inequalities is obtained. The magnitude of the violation increases as $E_{0} / \Phi$ decreases and as $D$ increases. It reaches the maximum 4 just like our simple model of Section 2 did, which is hardly surprising given the simplicity of the model.

Note that the number of double counts, that is, the number of coincidence counts between two detectors at the same station (either Alice or Bob), is a serious constraint on the combination of parameters $E_{0} / \Phi$ and $D$ that can be considered acceptable. Operating away from the region where an apparent violation of Bell inequalities arises 
a)

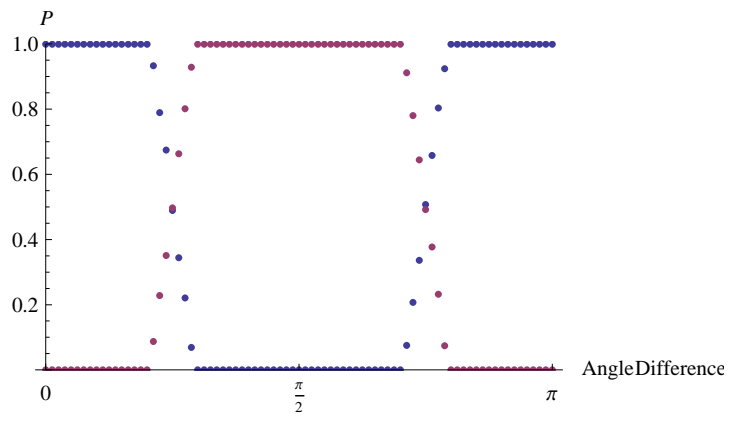

b)

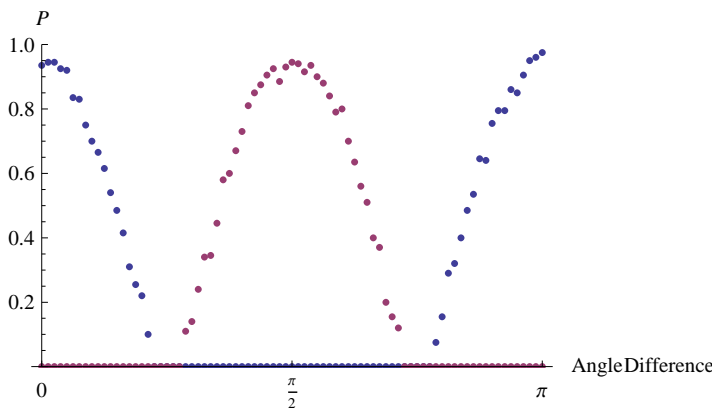

Figure 3: An actual detection probability as a function of the difference $(\lambda-\varphi)$. a) for Alice with $\frac{E_{0}}{\Phi}=2.3$, b) for Bob with $\frac{E_{0}}{\Phi}=1.9$. In both cases, the discriminator threshold was set to $D_{\text {threshold }}=0.3$ (with collection efficiency and gain set to 1). The pattern is similar to the Gisin-Larsson model [28, 27, 29].

a)

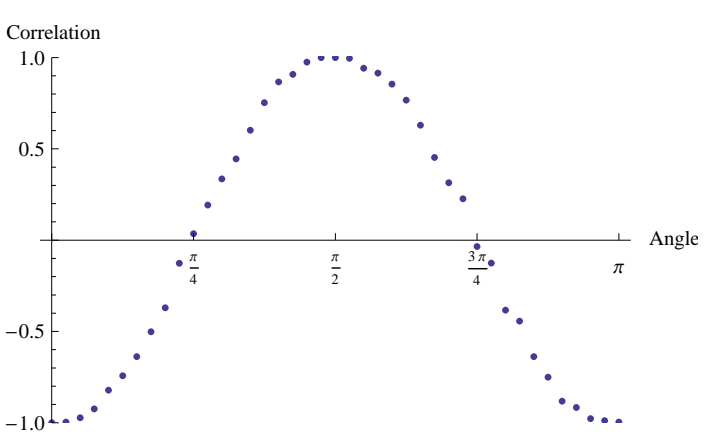

b)

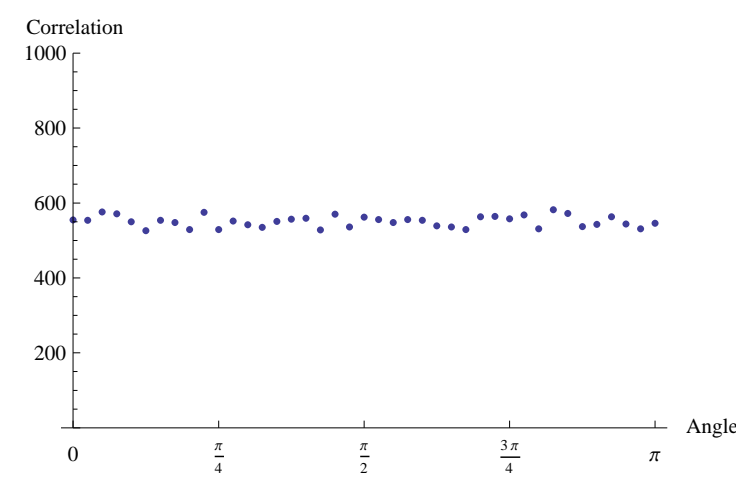

Figure 4: Measured results with the settings of Fig. 3, a) The correlation function is in excellent agreement with the $\cos (2 \Delta \phi)$ predicted by Quantum Mechanics. b) Total number of coincidences

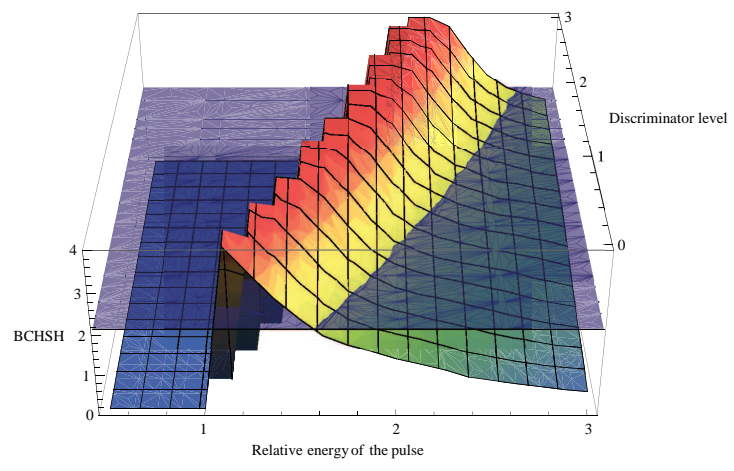

a)

b)

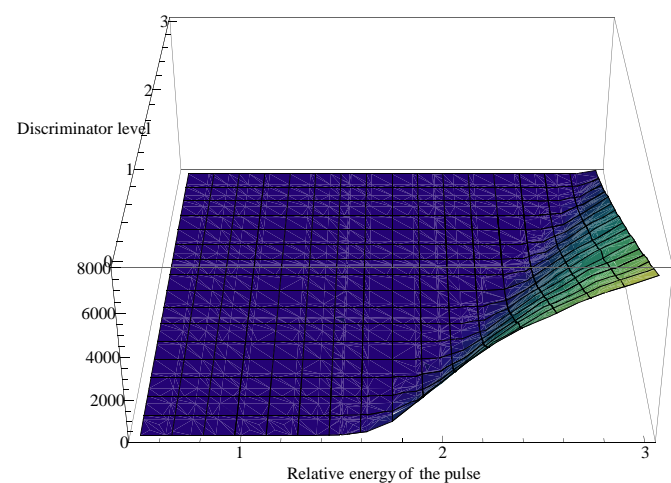

Figure 5: a) (Apparent) violation of Bell inequalities as a function of the relative energy of the pulse $E_{0} / \Phi$ and of the discrimination threshold $D$ (horizontal coordinates). The flat semi transparent horizontal layer at $\mathrm{BCHSH}=2 \mathrm{rep}-$ resents Bell inequalities. An apparent violation is observed for a combination of the two threshold.b) Corresponding Number of double counts. Having coincidence clicks between the + and - channel at each station should be avoided to remain in the 'single photon regime'. One is thus necessarily driven to regions where an apparent violation of Bell inequalities is observed. 
from the combined threshold cannot be done without having double counts appearing (see Fig. 5b). Just like in our simple model of Section 2 the constraint of having no double counts necessarily leads to an apparent violation of Bell inequalities.

We have performed more complex simulations with the implementation of various sources of noise, losses and fluctuations [47]. The fundamental result was unchanged. Naturally the noisier the simulation, the lesser the apparent violation of Bell inequalities. Introducing some multiplication noise and some dark counts diminishes the maximum observable, or at the very least it is only with a rather high discriminator threshold $D$ that such high values can be obtained. It also naturally increases the number of small output pulses, and thus necessitates to keep the discriminator threshold above a certain level to suppress the double counts, a level that is always concomitant with an apparent violation of Bell inequalities.

\section{Discussion}

The result of the simulation depends crucially on the classical behavior of a pulse at a polarizing beam splitter. We have indeed assumed throughout this article that pulses can be treated classically. At a PBS oriented along $\phi$, the energy of the pulse linearly polarized along $\lambda$ is split in both arms depending on $(\lambda-\phi)$, according to Malus law. It should be noted that the assumption that the field would be classically split at a PBS need not be in contradiction with known experimental facts, since many phenomena can be explain without a field quantization. For instance, it it well known that the photoelectric effect can be explained without resorting to the concept of photon[39], by considering classical fields and quantized energy levels in atoms [40]. In fact, it looks like that the only type of effects that are considered to require such a field quantization are of a similar type as the EPR-Bell experiments, which, as we have seen, can actually be explained with threshold detectors and classical fields.

The other possibility is of course to consider that each pulse contains one indivisible quantum of light (photon), each of them being either fully reflected or fully transmitter at a PBS, the which-way information being determined by Malus law, this time understood as a probability rule. In such a case, regardless of $\lambda$ and $\phi$, the detector always sees the same energy in the single photon regime, because a photon is either completely transmitted or completely reflected[48, 49, 50, 51], so that no sample selection bias can arise. It means that the role of the thresholds as a possible source of unfair sampling can only be neglected in an EPR-Bell setup if one assumes that the light impinging on a polarizing beam splitter is constituted of indivisible particles (light quanta). The assumption of fair sampling would thus be an assumption on the indivisibility of light quanta in disguise. This is usually considered the only possible interpretation of the anticorrelation experiments at a beam splitter [52, 53], but we would like to stress that this result can be enforced and reproduced classically, as soon as a detection threshold are considered, as was is shown above with Eqs. (3) and (4).

\section{Conclusion}

We have seen that the role of thresholds in photomultipliers and avalanche photodiodes is fundamental for the interpretation of EPR-Bell experiments, but this should also be true for other non-classical experiments in which the only difference between the classical case and the quantum case is the visibility of the interference effect, as was shown to be the case for all type of two-photon experiments by Klyshko [54, 55]. Indeed, as we have shown here in the case of EPR-Bell experiments, the introduction of threshold detectors in a classical framework has the simple effect of lowering the visibility of the interferences that would be obtained without such thresholds, that is, with detectors that would offer a linear response regardless of the incoming signal. Fair sampling should therefore no longer remain as an unproven assumption in nonclassical experiments, it is high time for this fundamental loophole to be thoroughly tested experimentally [56] with the same attention and care that has been devoted to the communication loopholes.

\section{References}

[1] J S Bell. Physics, 1:195, 1964.

[2] Luigi Accardi. Topics in quantum probability. Phys. Rep., 77:169-192, 1981.

[3] Luigi Accardi. The wave-particle dualism. A tribute to Louis de Broglie on his 90th Birthday, pages pp. 47-55. D. Reidel Publ. Company,Dordrecht, 1970. 
[4] Luigi Accardi and Andrei Khrennikov. Proc. of Foundations of Probability and Physics-4, volume 889 of AIP Conference Proceedings. AIP, New York, 2007.

[5] Karl Hess and Walter Philipp. Proc Natl Acad Sci U S A., 101(7):17991805., 2004.

[6] Karl Hess and Walter Philipp. Europhys. Lett., 57:775781, 2002.

[7] Andrei Yu. Khrennikov. J. Math. Phys., 44(6):2471- 2478, 2003.

[8] Andrei Yu. Khrennikov. Phys. Lett. A, 316:279-296, 2003.

[9] Andrei Yu. Khrennikov. Annalen der Physik, 12(10):575-585, 2003.

[10] Andrei Yu. Khrennikov. arxiv:quant-ph/0211073. 2002.

[11] Andrei Yu. Khrennikov. Non-Archimedean analysis: quantum paradoxes, dynamical systems and biological models. 1997.

[12] Andrei Yu. Khrennikov. J. of Math. Physics, 41(4):1768-1777, 2000.

[13] Andrei Yu. Khrennikov. Il Nuovo Cimento, B 115(2):179-184, 1999.

[14] Andrei Yu. Khrennikov. Interpretations of Probability, VSP Int. Sc. Publishers, Utrecht/Tokyo, 1999.

[15] A. Yu. Khrennikov. The principle of supplementarity: A contextual probabilistic viewpoint to complementarity, the interference of probabilities, and the incompatibility of variables in quantum mechanics. Foundations of Physics, 35(10):1655 - 1693, 2005.

[16] A Aspect, J Dalibard, and G Roger. Phys. Rev. Lett., 49:91, 1982.

[17] Gregor Weihs, Thomas Jennewein, Christoph Simon, Harald Weinfurter, and Anton Zeilinger. Phys. Rev. Lett., 81(23):5039, 1998.

[18] Thomas Scheidl, Rupert Ursin, Johannes Kofler, Sven Ramelow, Xiao-Song Ma, Thomas Herbst, Lothar Ratschbacher, Alessandro Fedrizzi, Nathan Langford, Thomas Jennewein, and Anton Zeilinger. Violation of local realism with freedom of choice, quant-ph:arxiv:0811.3129, 2008.

[19] M A Rowe, D Kielpinski, V Meyer, C A Sackett, W M Itano, C Monroe, and D J Wineland. Experimental violation of a bell's inequality with efficient detection. Nature, 409:791, 2001.

[20] Nils Bohr. Phys. Rev., 48:696-702, 1935.

[21] A Einstein, B Podolsky, and N Rosen. Phys. Rev., 47:777, 1935.

[22] P Pearle. Phys. Rev. D, 2:1418, 1970.

[23] John F. Clauser and Abner Shimony. Rep. Prog. Phys., 41:1881-1927, 1978.

[24] A Garrucio and V Rapisarda. Nuovo Cimento, A65:269, 1981.

[25] Anupam Garg and N. D. Mermin. Detector inefficiencies in the einstein-podolsky-rosen experiment. Phys. Rev. D, 35(12):3831-3835, Jun 1987.

[26] E. Santos. Phys. Lett. A., 212:10, 1996.

[27] Jan-Åke Larsson. Phys. Rev. A, 57(5):3304, 1998.

[28] N. Gisin and B. Gisin. Phys. Lett. A., 260:323, 1999.

[29] Jan-Åke Larsson. Quantum Paradoxes, Probability Theory, and Change of Ensemble. PhD thesis, Linköping Univ. Press, Sweden, 2000.

[30] L Pappalardo and V Rapisarda. Lett. Nuovo Cimento, 29:221, 1980.

[31] Caroline H. Thompson and Horst Holstein. The 'chaotic ball' model,local realism and the bell test loopholes, arxiv.org:quant-ph/0210150, 2002. 
[32] G. Adenier. Quantum entanglement, fair sampling, and reality: Is the moon there when nobody looks? American Journal of Physics, 76:147-152, February 2008.

[33] W. D. Oliver E. Diamanti E. Waks, K. Inoue and Y. Yamamoto. Selected Topics in Quantum Electronics, 9(6):1502-1511, 2003.

[34] R.J. McIntyre. Comparison of photomultipliers and avalanche photodiodes for laser applications. IEEE Transactions on Electron Devices, 17(4):347-352, 1970.

[35] S.D. Personick. Receiver design for optical fiber systems. Proceedings of the IEEE, 65(12):1670-1678, 1977.

[36] Hollenhorst J.N. Fundamental limits on optical pulse detection and digital communication. Lightwave Technology, Journal of, 13(6):1135-1145, 1995.

[37] Brunner Nicolas, Branciard Cyril, and Gisin Nicolas. Can one see entanglement ?, arxiv:0802.0472v1. 2008.

[38] G. Weihs et al. "quantum physics from A to Z", arxiv.org:quant-ph/0505187. 2005.

[39] Willis E. Lamb. Appl. Phys., B66:77-84, 1995.

[40] W. E. Lamb and M. O. Scully. in Polarization, matter and radiation, pages 363-369. Presses Univ. de France, Paris, 1969.

[41] Photomultiplier tubes : Principles and applications. Technical report, Photonis, Brive, France, 2002.

[42] Photomultiplier handbook. Technical report, Burle Technologies, Lancaster, Pennsylvania, 1980.

[43] Glenn F. Knoll. Radiation Detection and Measurement. Wiley \&Sons, 1999.

[44] A. G. Wright. Technical Reprint R/P083, Electron Tubes Ltd., 2007.

[45] Alain Aspect. Trois tests expérimentaux des inégalités de Bell par mesure de corrélation de polarisation de photons. No. 2674, Université de Paris-Sud, Centre D’Orsay, 1983.

[46] Philippe Grangier. Etude expérimentale de propriétés non-classiques de la lumière: interférence à un seul photon. PhD thesis, Université de Paris-Sud, Centre D’Orsay, 1986.

[47] G. Adenier. Local Realist Approach and Numerical Simulations of Nonclassical Experiments in Quantum Mechanics. PhD thesis, Vaxjo University, 2008.

[48] P. A. M. Dirac. The Principles of Quantum Mechanics. Clarendon Press, Oxford, 1958.

[49] Caslav Brukner and Anton Zeilinger. acta physica slovaca, 49(4):647-652, 1999.

[50] Caslav Brukner and Anton Zeilinger. in "Time, Quantum, Information”. Springer, 2003.

[51] Whooters. Phys Rev D, 23:357, 1981.

[52] G. Roger P. Grangier and A. Aspect. Europhys. Lett., 1:173, 1986.

[53] Jacques Vincent, Wu E, Grosshans Frederic, Treussart Francois, Grangier Philippe, Aspect Alain, and Roch Jean-Francois. Experimental realization of wheeler's delayed-choice gedanken experiment. Science, 315(5814):966-968, 2007.

[54] D.N. Klyshko. Two-photon (squeezed) light: Classical and quantum effects. Physics Letters A, 146(3):93-101, 1990.

[55] D.N. Klyshko. Observable signs of nonclassical light. Physics Letters A, 213(1-2):7-15, 1996.

[56] G. Adenier. Journal of Russian Laser Research, 29(5):409, 2008. 